\title{
Direct visualization of ageing in colloidal glasses
}

\author{
Rachel E Courtland and Eric R Weeks ${ }^{1}$ \\ Physics Department, Emory University, Atlanta, GA 30322, USA \\ E-mail: weeks@physics.emory.edu
}

Received 28 October 2002

Published 16 December 2002

Online at stacks.iop.org/JPhysCM/15/S359

\begin{abstract}
We use confocal microscopy to directly visualize the dynamics of ageing colloidal glasses. We prepare a colloidal suspension at high density, a simple model system that shares many properties with other glasses, and initiate experiments by stirring the sample. We follow the motion of several thousand colloidal particles after the stirring and observe that their motion significantly slows as the sample ages. The ageing is both spatially and temporally heterogeneous. Furthermore, while the characteristic relaxation timescale grows with the age of the sample, nontrivial particle motions continue to occur on all timescales.
\end{abstract}

\section{Introduction}

Colloidal suspensions have long been used as model systems to study the glass transition [1-5]. As the concentration of a colloidal suspension is increased, the motion of the colloidal particles becomes increasingly slowed. The glass transition is considered to occur at the concentration at which particle motion ceases to be diffusive on long timescales [5]. Recent work has studied the behaviour of simple colloidal suspensions as this transition is approached [6-8]. In this paper we examine the behaviour of glassy samples. Unlike that of supercooled colloidal fluids, the behaviour of a colloidal glass depends on the history of the sample: particle motion becomes increasingly slowed as the sample ages [9-13]. We use confocal microscopy to study the three-dimensional motions of colloidal particles in an ageing colloidal glass. We find that ageing occurs due to slight rearrangements that are both spatially and temporally heterogeneous. Moreover, even after the sample has aged for long times, small but significant motions occur on short timescales. Previous work has seen similar evidence of spatial and temporal heterogeneities in other ageing systems [9, 16-19].

\footnotetext{
1 Author to whom any correspondence should be addressed. 


\section{Experimental methods}

We use poly(methyl methacrylate) (PMMA) particles of radius $a=1.18 \mu \mathrm{m}$ and polydispersity $\sim 5 \%$, sterically stabilized by a thin layer of poly-12-hydroxystearic acid. The particles are dyed with rhodamine and suspended in a mixture of organic solvents (cyclohexylbromide and decalin) that closely matches both the density and index of refraction of the particles. We use a scanning laser confocal microscope to acquire images of a viewing volume of $63 \mu \mathrm{m} \times 58 \mu \mathrm{m} \times 12 \mu \mathrm{m}$ at the rate of three images per minute. The viewing volume typically contains $\sim 2400$ particles. We focus at least $60 \mu \mathrm{m}$ away from the cover slip of the sample chamber to avoid wall effects. We identify particles with a horizontal accuracy of $0.03 \mu \mathrm{m}$ and a vertical accuracy of $0.05 \mu \mathrm{m}$, and track them in three dimensions over the course of the experiment $[14,15]$.

The control parameter for colloidal phase behaviour is the sample volume fraction $\phi$. While the rhodamine imparts a slight charge upon the particles, their phase behaviour, $\phi_{\text {freeze }}=0.38$ and $\phi_{\text {melt }}=0.42$, is similar to that of hard spheres $\left(\phi_{\text {freeze }}=0.494\right.$ and $\left.\phi_{\text {melt }}=0.545\right)$. We observe a glass transition at $\phi_{g} \approx 0.58$, in agreement with what is seen for hard spheres $[1,8]$. We examined samples with volume fractions in the range $\phi \approx 0.58-0.62$. These samples form small crystals which nucleate at the cover slip, but do not form crystals within the bulk of the sample even after several weeks.

To initialize the system, a small length of wire is inserted into each sample chamber. After placing a sample on the microscope stage, a hand-held magnet is used to pull and rotate the wire through the sample for several min. The subsequent particle dynamics is reproducible after this stirring. Within a minute of ending the stirring, transient flows within the sample greatly diminish, and the particles move slowly enough to be identified and tracked. This defined our initial time $t=0$ for each sample, although the results below are not sensitive to variations of this choice. During the experiments, no crystallization was observed within the viewing volume. Note that in many ageing studies, the initial sample is prepared by a temperature quench (corresponding to a rapid increase of the volume fraction in our experiments). The initial conditions in our experiments correspond to a shear-melted sample; the volume fraction remains constant.

\section{Results}

As a glass ages, we expect dynamical properties to change with time. To investigate this, we consider small temporal portions of a data set and calculate the mean square displacement (MSD) $\left\langle\Delta r^{2}\right\rangle$ for each portion, where the angle brackets indicate an average over all particles and all initial times within each temporal portion. Figure 1 displays the progression of the MSD curves of a colloidal glass $(\phi=0.62)$ over a period of $2 \mathrm{~h}$, showing a marked change as the sample ages. The data is divided logarithmically into segments according to the sample age $t_{w}$, the time waited since stirring, such that $t_{w}^{n+1}=c t_{w}^{n}$, where $c=1.4$, $t_{w}^{0}=3.0 \mathrm{~min}$, and $n=[1,2, \ldots, 11]$. The MSD for a given $t_{w}$ is calculated using data within the interval $\left[c^{-1} t_{w}, c t_{w}\right]$. The MSD behaviour at low and intermediate lag times is consistent with that of supercooled liquids [6,7]. For $\Delta t<30 \mathrm{~s}$ (not shown), the MSD grows roughly linearly with $\Delta t$, corresponding to the diffusion of particles inside 'cages' formed by neighbouring particles [5,6]. The MSD at intermediate lag times exhibits a plateau, as particles are locally confined by their neighbours. At larger lag times, the MSD curves each show a slight upturn, reminiscent of supercooled liquids [13]. For supercooled liquids, this upturn corresponds to cage rearrangements: the configuration of particles around a caged particle changes noticeably [6]. In our ageing samples, we see similar, though smaller, motions, and the upturn in the MSD is less obvious than that seen for liquids. These motions, which 


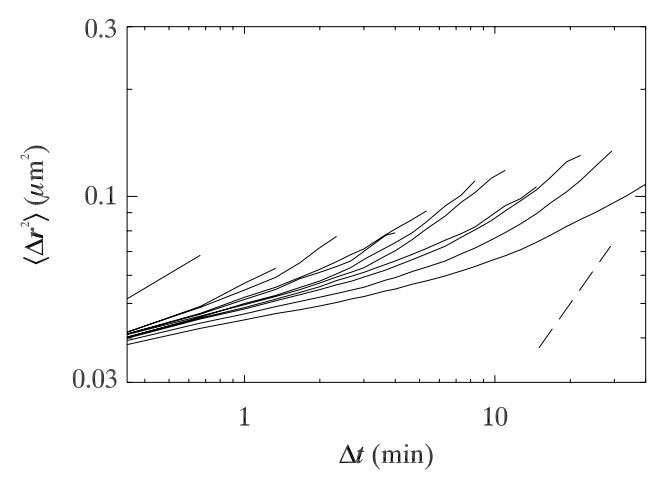

Figure 1. MSD versus lag time for a colloidal glass $(\phi \approx 0.62)$. The total length of the data set is $120 \mathrm{~min}$. Data are divided logarithmically according to sample age. The geometric mean ages of the curves, from left to right, are $\overline{t_{w}}=3.0,4.2,5.9,8.2,12,16,23,32,44,62,87 \mathrm{~min}$. The dashed line has a slope of 1 .

change the local positions of particles, are probably related to cage rearrangements $[12,13]$. Presumably these local rearrangements lead to the ageing of the sample. However, unlike the case for supercooled liquids, the timescale for the upturn in the MSD depends strongly on the age of the sample, $t_{w}$, as is clear from figure 1 [13]. For larger values of $t_{w}$, the plateau extends over a larger range of $\Delta t$. This behaviour has been seen before in a variety of ageing systems $[10,11]$.

We observe also that the MSD of a colloidal glass does not evolve uniformly. Consider the four rightmost curves in figure 1 , corresponding to $\overline{t_{w}}=32,44,62$, and $87 \mathrm{~min}$. The first two curves $\left(\overline{t_{w}}=32,44 \mathrm{~min}\right)$ are virtually indistinguishable, showing that the dynamics of particles within the imaging volume has not changed. However, the next two curves $\left(\overline{t_{w}}=62\right.$, $87 \mathrm{~min}$ ) are quite different, showing that these particles have aged. This suggests that ageing in our small observation volume is in part temporally heterogeneous. It is to be expected that if a larger volume were examined, as in light scattering experiments, the dynamics would appear to evolve more smoothly with $t_{w}[10,11]$, although recent techniques which avoid a temporal averaging also show heterogeneous dynamics [19].

The temporal averaging used to calculate the MSD obscures some of the details of this temporal heterogeneity. However, it is unclear how to best study the dynamics given that the timescales for such dynamics may vary dramatically with the sample age $t_{w}$. Furthermore, the mobile particles responsible for the upturn in the MSD are only moving slightly further than the immobile particles; the latter still move locally due to their Brownian motion. To help distinguish the rearranging particles from those which only move within their cage, we average the trajectory of each particle over $1 \mathrm{~min}$. The results that follow are not sensitive to the choice of this averaging time.

To investigate the nature of the temporally heterogeneous ageing, we choose a fixed lag time $\Delta T$ and study how the dynamical behaviour changes with $t_{w}$. We might expect that for $t_{w} \ll \Delta T, t_{w} \approx \Delta T$, and $t_{w} \gg \Delta T$, we should see strikingly different behaviours. We choose $\Delta T=10$ min to allow us to resolve these three regimes with our data. The solid curve in figure 2(a) shows $\left\langle\Delta x^{2}\right\rangle$ plotted as a function of sample age, $t_{w}$; here, the angle brackets do not indicate a time average, but only a particle average, unlike for the MSD. As $t_{w}$ increases, $\left\langle\Delta x^{2}\right\rangle$ generally decreases, which is not surprising given the behaviour shown in figure 1: at large $t_{w}$, particle motion on a timescale of $\Delta T=10 \mathrm{~min}$ reflects the motion of particles confined by their neighbours. However, in addition to this overall relaxation of $\left\langle\Delta x^{2}\right\rangle$, 

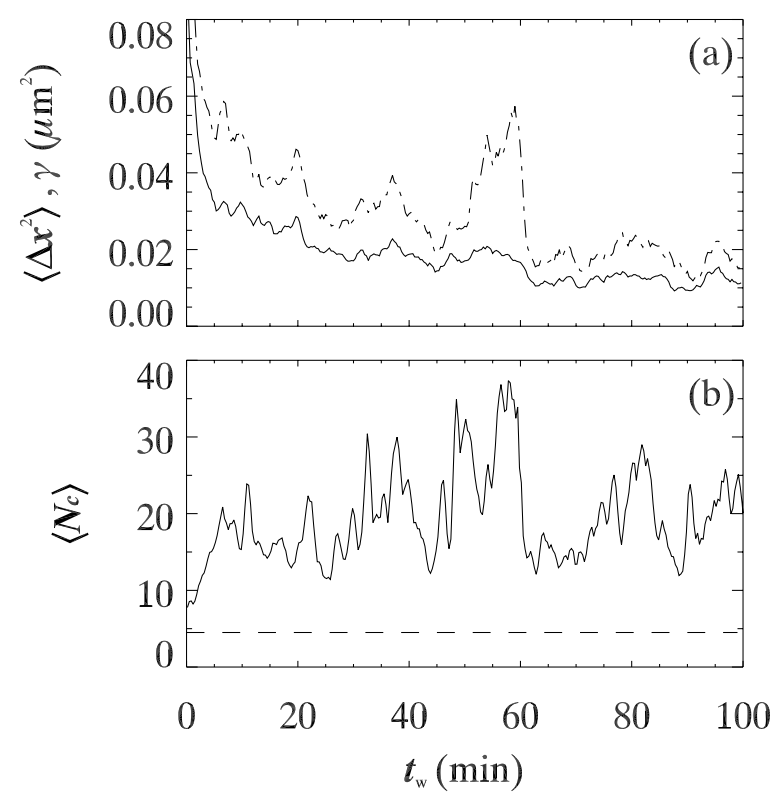

Figure 2. Various ensemble features as a function of sample age, $t_{w}$. The time lag is fixed $(\Delta T=10 \mathrm{~min})$ and the data are the same as those shown in figure 1. (a) $\left\langle\Delta x^{2}\right\rangle$ and $\gamma=\sqrt{\left\langle\Delta x^{4}\right\rangle / 3}$ (dashed). (The $x$-component is used, as the $z$-component is noisier due to optical effects.) (b) The average number of particles in a cluster of mobile particles; see the text for details. The dashed line indicates the average cluster size if mobile particles were randomly distributed.
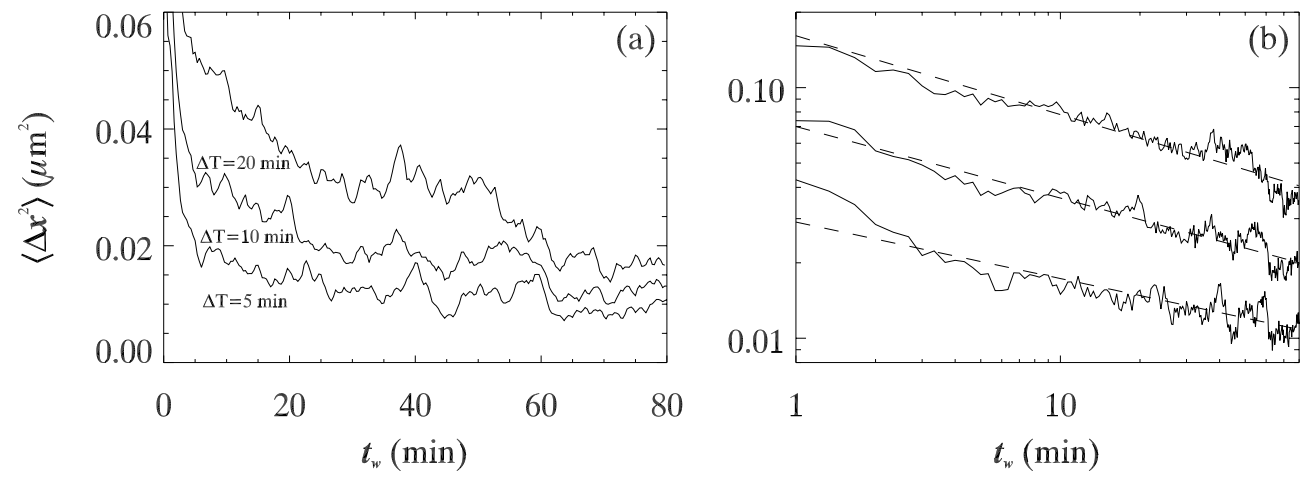

Figure 3. $\left\langle\Delta x^{2}\right\rangle$ as a function of sample age for various fixed values of lag time, $\Delta T$, on linear axes (a) and log-log axes (b). The straight lines in (b) are fits to power law decay, $\left\langle\Delta x^{2}\right\rangle \sim t_{w}{ }^{-a}$, with $a=0.23,0.29$, and 0.31 for $\Delta T=5,10$, and $20 \mathrm{~min}$, respectively. The curves in (b) corresponding to $\Delta T=5$ and $20 \mathrm{~min}$ have been vertically offset for clarity. The data are the same as in the previous figures.

figure 2(a) shows a number of abrupt events, when $\left\langle\Delta x^{2}\right\rangle$ jumps in value before relaxing further. Further confirmation of the temporally heterogeneous nature of this process is demonstrated by the dashed curve in figure 2(a), which shows $\gamma=\sqrt{\left\langle\Delta x^{4}\right\rangle / 3}$. If the dynamics was purely Gaussian, i.e. diffusive, we would expect $\gamma=\left\langle\Delta x^{2}\right\rangle$. The consistently higher value of $\gamma$ and its prominent correlation with events in $\left\langle\Delta x^{2}\right\rangle$ indicate that the ensemble dynamics is highly non-Gaussian and events in $\left\langle\Delta x^{2}\right\rangle$ particularly so. 

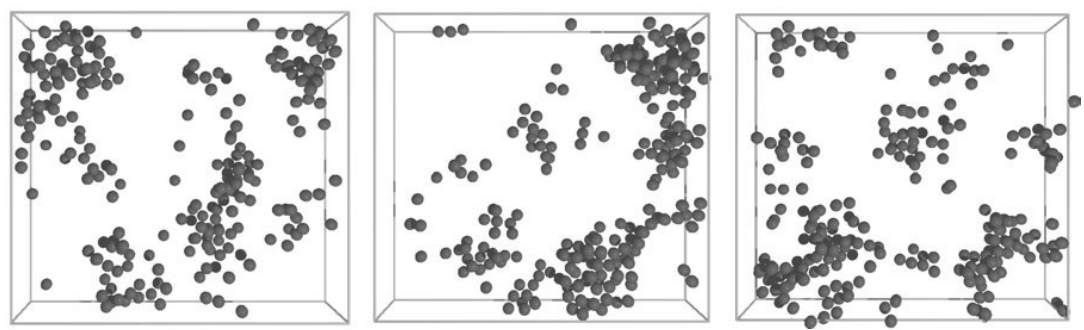

Figure 4. Locations of the $10 \%$ most mobile particles at three different ages $t_{w}$. For each picture, mobility was determined by calculating displacements $\Delta r$ over an interval $\left[t_{w}, t_{w}+\Delta T\right]$, with $\Delta T=10 \mathrm{~min}$. Left: $t_{w}=10 \mathrm{~min}$, and $\Delta r>0.43 \mu \mathrm{m}$ for the most mobile particles. Middle: $t_{w}=55 \mathrm{~min}, \Delta r>0.34 \mu \mathrm{m}$. Right: $t_{w}=95 \mathrm{~min}, \Delta r>0.33 \mu \mathrm{m}$. The data are the same as shown in previous figures, and the choices of $t_{w}$ correspond to local maxima of $\gamma$ in figure 2(a). The particles are drawn to scale $(2.36 \mu \mathrm{m}$ diameter $)$ and the box shown is the entire viewing volume (within a much larger sample chamber).

It is intriguing that there is no unique behaviour for $t_{w} \approx \Delta T$; instead, ageing 'events' occur at many different values of $t_{w}$. To further confirm this, we plot $\left\langle\Delta x^{2}\right\rangle$ versus sample age for several different values of $\Delta T$ in figure 3. The curves are clearly related; many events are simultaneously present in all curves. In figure 3(b) these curves are plotted on log-log axes, showing that the overall decrease in $\left\langle\Delta x^{2}\right\rangle$ is consistent with a power law decay, $\left\langle\Delta x^{2}\right\rangle \sim t_{w}^{-a}$. For different samples and different choices of $\Delta T$, we find that $a$ ranges between 0.05 and 0.5 .

For supercooled colloidal liquids it is known that the dynamics is spatially heterogeneous as well as temporally heterogeneous $[7,8]$. To investigate this for our ageing samples, we plot the 3D locations of mobile particles in figure 4 for $t_{w}=10,55$, and $95 \mathrm{~min}$. The mobility of a particle is defined as its displacement during the interval $t_{w}$ to $t_{w}+\Delta T$ (with $\Delta T=10 \mathrm{~min}$ ). The particles shown are the $10 \%$ most mobile at those times and are generally grouped into large clusters. Varying thresholds does not substantially change these pictures. Our results are similar to the spatial heterogeneities seen in simulations of ageing [16]. Signs of heterogeneities have also been seen in ageing experiments which studied glycerol [17]. Previous microscopic studies of colloidal glasses did not show such large clusters [7]; we find that the slight averaging of the particle trajectories discussed above (over a 1 min interval) is necessary to distinguish the rearrangements from the local Brownian motion of caged particles. Without averaging, our pictures look similar to those of [7], and with averaging, the data of [7] look similar to the results shown here.

It is striking that the mobile particles are clustered for all values of $t_{w}$, notably at both $t_{w}=10 \mathrm{~min}=\Delta T$ and $t_{w}=95 \mathrm{~min} \gg \Delta T$. We investigate the $t_{w}$-dependence of the spatial clustering of mobile particles by generating similar pictures for each $t_{w}$ and measuring the average size of the clusters in each picture. (A cluster is defined as all connected mobile particles which are closer to each other than $3.2 \mu \mathrm{m}$, the first minimum of $g(r)$, the pair correlation function.) Again, mobile particles are defined as the particles with displacements in the top $10 \%$ at each $t_{w}$, using $\Delta T$ to characterize the displacements. The average cluster size shows large fluctuations, as seen in figure 2(b). If the $10 \%$ most mobile particles were randomly distributed in space, the average cluster size would be $\sim 4.5$ (dashed line in figure 2(b)); however, we find that the average cluster size is always larger than this value. The larger average cluster sizes correspond to locally large values of $\left\langle\Delta x^{2}\right\rangle$ and $\gamma$, seen in figure 2(a).

Our most surprising result is that overall, the cluster sizes do not increase dramatically with $t_{w}$. For the sample to age, particles must move, and may do so in small groups such as these clusters. For early ages $t_{w}$, it might be expected that particle rearrangements may involve very 
few particles, which can rearrange relatively quickly, thus causing the MSD to have a shorter plateau. For larger $t_{w}$, if the rearrangements involved more particles, this could be more difficult and take longer to occur, thus explaining the slower dynamics reflected in the later MSD curves of figure 1. However, this is not observed. As seen in figure 2(b), the average cluster size does not dramatically change as a function of $t_{w}$. This remains true even if we scale the choice of $\Delta T$ with $t_{w}$ (not shown). Thus, it is not yet clear what ultimately causes the dynamics to slow as our sample ages. While the rearrangements occur via groups such as those shown in figure 4, we have not found anything in their character which changes as the sample ages.

While all the data shown in the figures correspond to one sample with $\phi=0.62$, we have investigated five other samples in the range $0.58 \leqslant \phi \leqslant 0.62$. All data sets show similar behaviour, and as yet we see no clear $\phi$-dependence in our results.

\section{Conclusions}

Ageing in our colloidal samples appears to be due to the most mobile particles in the sample, seen in figure 4 and reflected by the fluctuations in figure 2 . In order for the sample to age, particles must move to new positions. Our results show that such rearrangements are both spatially and temporally heterogeneous. In general, each ageing event appears spatially uncorrelated with the previous one. The surprising result is that significant fluctuations occur on timescales $\Delta T \ll t_{w}$, despite the fact that for large $t_{w}$, the MSD shows only a plateau at such $\Delta T$ (see figure 1). We find, in fact, that the plateau in the MSD is due to a temporal average over these infrequent rearrangement events and the more prevalent caged behaviour. We have also attempted to investigate the behaviour of the most immobile particles, but this has been difficult, as every particle still exhibits nontrivial Brownian motion, and there is no unambiguous way to distinguish the most immobile particles.

Thus, the changing character of the MSD (figure 1) and the related decay in $\left\langle\Delta x^{2}\right\rangle$ for a fixed $\Delta T$ (shown in figure 3 ) seem to be the most significant dynamical changes in ageing colloidal glasses. In our future work, we plan to investigate the microscopic structural changes associated with these dynamical changes.

\section{Acknowledgments}

We thank J-P Bouchaud and S Franklin for helpful discussions. This work was supported by NASA and the University Research Committee of Emory University.

\section{References}

[1] Pusey P N and van Megen W 1986 Nature 320340

Pusey P N and van Megen W 1987 Phys. Rev. Lett. 592083

[2] Bartsch E, Frenz V, Möller S and Silescu H 1993 Physica A 201363

[3] van Megen W and Underwood S M 1994 Phys. Rev. E 494206

[4] van Blaaderen A and Wiltzius P 1995 Science 2701177

[5] Angell C A 2000 J. Phys.: Condens. Matter 126463 Ediger M D, Angell C A and Nagel S R 1996 J. Phys. Chem. 10013200

[6] Weeks E R and Weitz D A 2002 Phys. Rev. Lett. 89095704

[7] Weeks E R, Crocker J C, Levitt A C, Schofield A and Weitz D A 2000 Science 287627

[8] Kegel W K and van Blaaderen A 2000 Science 287290

[9] Bouchaud J P 2000 Soft and Fragile Matter: Nonequilibrium Dynamics, Metastability and Flow ed M E Cates and M R Evans (Bristol: Institute of Physics Publishing) pp 285-304

[10] van Megen W, Mortensen T C, Williams S R and Müller J 1998 Phys. Rev. E 586073

[11] Cipelletti L, Manley S, Ball R C and Weitz D A 2000 Phys. Rev. Lett. 842275

[12] Bonn D, Tanaka H, Wegdam G, Kellay H and Meunier J 1998 Europhys. Lett. 4552 
[13] Knaebel A, Bellour M, Munch J-P, Viasnoff V, Lequeux F and Harden J L 2000 Europhys. Lett. 5273

[14] Dinsmore A D, Weeks E R, Prasad V, Levitt A C and Weitz D A 2001 Appl. Opt. 404152

[15] Crocker J C and Grier D G 1996 J. Colloid Interface Sci. 179298

[16] Vollmayr-Lee K, Kob W, Binder K and Zippelius A 2002 J. Chem. Phys. 1165158

[17] Miller R S and MacPhail R A 1997 J. Phys. Chem. B 1018635

[18] Castillo H B, Chamon C, Cugliandolo L F and Kennett M P 2002 Phys. Rev. Lett. 88237201

[19] Cipelletti L, Bissig H, Trappe V, Ballesta P and Mazoyer S 2003 J. Phys.: Condens. Matter 15 S257 